\title{
EXISTENCE FOR CONSTRAINED DYNAMIC GRIFFITH FRACTURE WITH A WEAK MAXIMAL DISSIPATION CONDITION
}

\author{
GIANNI DAL MASO, CHRISTOPHER J. LARSEN, AND RODICA TOADER
}

\begin{abstract}
The study of dynamic fracture is based on the dynamic energy-dissipation balance. It is easy to see that this condition is always satisfied by a stationary crack together with a displacement satisfying the system of elastodynamics. Therefore to predict crack growth a further principle is needed. In this paper we introduce a weak maximal dissipation condition that, together with elastodynamics and energy balance, provides a model for dynamic fracture, at least within a certain class of possible crack evolutions. In particular, we prove the existence of dynamic fracture evolutions satisfying this condition, subject to smoothness constraints, and exhibit an explicit example to show that maximal dissipation can indeed rule out stationary cracks.
\end{abstract}

Keywords: Wave equation, dynamic fracture mechanics, cracking domains.

2010 Mathematics Subject Classification: 35L05, 35L20, 26B30, 74J05, 74R10.

\section{INTRODUCTION}

A long term goal of the mathematical analysis of dynamic fracture is to formulate and study precise models for crack growth when inertial effects are taken into account. As in the quasistatic case (see [3] and the references therein), it would be desirable to consider cracks with arbitrary shape and topology. This general framework could be used to predict a behavior such as kinking and branching, without additional hypotheses. These models should be based on as few general principles as possible, determined by the basic physics of the problem. The precise formulation of these principles should lead to a rigorous mathematical proof of all details of the models.

Let us mention that the existence of solutions satisfying all conditions considered in a specific model is a primary issue. This is not just a mathematical luxury - a formulation that prescribes too many properties runs a strong risk of not having solutions. The proof of the existence of a solution in the framework of a model, under suitable assumptions on the data, guarantees that this model has no internal contradictions. Only in this case one can use it to compute approximate solutions and then compare the predictions of the model with the outcome of experiments.

Several results have already been obtained in fracture mechanics, starting from the seminal paper by Griffith [11], who established an energy criterion for crack stability: a crack can grow only if the elastic energy released when it grows is larger than or equal to the energy spent to produce the new portion of the crack. The infinitesimal version of this principle leads to the notions of energy release rate and of toughness. In the two-dimensional case, the former is defined as the elastic energy released per unit length of new crack, and depends on the deformation of the body and on the geometry of the crack, while the latter is a material property, defined as the energy per unit length dissipated in the process of crack production. These notions, which nowadays are the basis of fracture mechanics, where introduced by Irwin [14], who also showed the relationship between energy release rate and stress intensity factors in the case of linear elasticity. 
Mott [18] extended Griffith's criterion to the case of dynamic fracture, taking the kinetic energy into account. This leads to the dynamic energy-dissipation balance: the sum of the kinetic energy and of the elastic energy at time $t$, plus the energy dissipated by the crack between time zero and time $t$, is equal to the initial energy plus the total work done between time zero and time $t$.

The modern approach to dynamic fracture presented in the books $[10,5,17,21,20,1,22]$ is based on these ideas. The fracture criteria are expressed in terms of the dynamic energy release rate, which is connected with the dynamic stress intensity factors. Besides the energy dissipated for crack production, these books take also into account further sources of dissipation due to thermal effects and to the speed of the crack process.

Note, however, that the dynamic energy-dissipation balance is not sufficient to determine the evolution of a crack, since elastodynamics with a stationary crack will always satisfy energy balance. This shows that there is a need for an additional principle for crack growth.

Recently, a phase-field approach has been developed, for which existence has been shown (see $[3,4,2,16,13])$. The crack is replaced by a phase-field approximation: a function $v$ which takes the value 0 near the crack and the value 1 far from it. In these models, an energy minimization condition on $v$ provides a principle that can require the crack to grow (so that stationary cracks are not always solutions).

Although this approach is very efficient from the numerical point of view, the convergence of the phase-field solutions to the solutions of the crack problem as the approximation parameter tends to 0 has been proved only in the quasistatic regime. Indeed, the correct form of the limiting problem, with a sharp crack instead of a regularized crack, is not yet completely clear. One possible principle to replace the minimality in the phase-field model is a maximal dissipation condition that does not require any crack regularity, proposed in [15].

The aim of this paper is to introduce a variant of this maximal dissipation condition and to show that, under suitable assumptions on the initial and boundary conditions, we can prove the existence of a solution to the dynamic crack problem with a prescribed crack path. This is defined as a crack-displacement pair such that the displacement satisfies the system of elastodynamics out of the crack set and the pair satisfies the dynamic energydissipation balance and the maximal dissipation condition. We also show that this condition is strong enough to prevent static cracks from being solutions in the case of certain initial and boundary conditions.

To our knowledge, this is the first mathematical proof of an existence result for dynamic fracture with sharp cracks. Since the interaction between elastodynamics and crack growth is a source of severe mathematical difficulties, we attack this problem in a simplified model, which still exhibits some of the relevant mathematical difficulties. The long term goal is to extend these results to the fully general problem - the dream is to avoid any assumptions about the regularity or any other properties of the crack set.

The model we consider here is linearly elastic with antiplane displacement. Therefore, the reference configuration is contained in the plane, the displacement $u$ is scalar, and the system of elastodynamics reduces to the scalar wave equation. The crack follows a sufficiently regular prescribed path. We consider only the problem of crack growth, assuming that an initial crack is already present. The issue of crack nucleation is out of the scope of this paper.

We neglect all thermal effects, as well as other sources of dissipation, except for the energy spent to produce new crack. Our point is that the main mathematical difficulties to obtain an existence result are already present in this simplified model, and that more realistic models could be studied later by adapting the ideas and techniques developed here.

As described in detail below, the crack evolutions we are considering are characterized by their length $s(t)$, considered as a function of time $t$. Given such a (sufficiently regular) function $s$, and appropriate initial and boundary data, to find the corresponding displacement 
$u$ we have to solve the wave equation off the time-dependent crack set, with zero Neumann condition on the crack.

This problem already exhibits a mathematical difficulty, due to the time dependence of the domains. The classical results on the wave equation in time-dependent domains cannot be applied directly, since they require suitable regularity assumptions on the boundary, which are clearly not satisfied by cracked domains.

The existence of a solution to this problem in domains with a prescribed growing crack was proved in [6] under very general assumptions, which allow for kinking and branching. The uniqueness, however, is an open problem in this general setting. Since in our treatment of the problem the uniqueness of the solution $u$ of the wave equation off the crack set is crucial, in our model with a prescribed crack path we assume more regularity on $s$ in order to apply the uniqueness result proved in [7]. In particular, we assume some uniform bounds on the first three time derivatives of $s$.

According to Mott's ideas, in our model we assume the dynamic energy-dissipation balance. Using the standard rule to compute the dynamic energy release rate for mode III cracks, it is possible to show that, under appropriate regularity assumptions, for every crack-displacement pair satisfying the dynamic energy-dissipation balance the dynamic stress intensity factor must be proportional to the toughness when the crack tip moves with a positive speed, with a universal proportionality constant. We will not develop this topic in this paper.

As remarked above, since a stationary crack and the corresponding solution to the wave equation will always satisfy the dynamic energy-dissipation balance, one needs to add a principle requiring that, in certain situations, the crack must grow. Principles of this kind are present, in an implicit or explicit way, in the above mentioned books on dynamic fracture. Since we want to use this principle to prove an existence result, we need a precise mathematical formulation.

In the thermodynamical approach to dynamic fracture, a principle of maximal dissipation rate was already used (see, e.g., [21] and [12]). In our context, the analysis of some phase-field models of dynamic fracture, studied in $[4,16]$, also led to a maximal dissipation principle, which was formulated in [15]. Since we do not take thermal effects into account, and the only dissipative mechanism in our model is crack production, our maximal dissipation is defined only in terms of the crack length.

The spirit of this maximal dissipation condition is simply that the crack must run as fast as possible, consistent with energy balance. Below we give a precise and equivalent version of that condition, when the crack path is prescribed and the crack at time $t$ is uniquely determined by its length $s(t)$.

First, we define a class of admissible evolutions, namely, those functions $s$ which, together with the corresponding displacement $u$, satisfy the dynamic energy-dissipation balance. An admissible evolution $s$ is said to satisfy maximal dissipation on $[0, T]$ if, for all $\tau \in[0, T]$, there is no admissible $\sigma$ such that $\sigma(t) \geq s(t)$ for $t \in[0, \tau]$ and $\sigma(\tau)>s(\tau)$.

For technical reasons, we are able to prove the existence of an admissible evolution satisfying the previous condition only in a quantitative way, depending on a prescribed threshold $\eta>0$. Up to additional regularity assumptions on $s$ and its competitors $\sigma$, this leads to the following definition: An admissible evolution $s$ is said to satisfy our $\eta$-maximal dissipation condition on $[0, T]$ if, for all $\tau \in[0, T]$, there is no admissible $\sigma$ such that $\sigma(t) \geq s(t)$ for $t \in[0, \tau]$ and $\sigma(\tau)>s(\tau)+\eta$.

For a given $\eta>0$, which can be interpreted as a characteristic length associated with the material, we propose $\eta$-maximal dissipation as a selection criterion among all crack evolutions which satisfy the dynamic energy-dissipation balance. Our main result, in Section 3 , is the existence of an $\eta$-maximal dissipation solution, for any prescribed initial and boundary conditions. 
Furthermore, in Section 4, we give an explicit example to show that this maximal dissipation condition is meaningful, in that if the elastic singularity of the initial data is large enough, it is possible to grow the crack while balancing energy. In particular, this shows that a stationary crack does not always satisfy $\eta$-maximal dissipation, and therefore it does not always satisfy maximal dissipation.

\section{NotATION AND PRELIMINARY RESUlts}

As mentioned in the introduction, we consider only linear elasticity with antiplane displacement. In this section we make precise the regularity assumptions on the data and on the solution of our problem. The function spaces used in the sequel are standard in the theory of partial differential equations. For their definition and properties we refer, e.g., to $[9]$.

Let $\Omega$ be a bounded open set in $\mathbb{R}^{2}$ with Lipschitz boundary $\partial \Omega$. We assume that the crack evolves along a prescribed simple curve $\Gamma$ of class $C^{2,1}$ contained in $\Omega$ except for its end-points, which belong to $\partial \Omega$. We also assume that $\Gamma$ divides $\Omega$ into two subsets $\Omega^{+}$ and $\Omega^{-}$, both having a Lipschitz boundary. This technical assumption excludes that $\Gamma$ is tangent to the boundary.

The hypothesis on the regularity of the boundary $\partial \Omega$ is satisfactory and includes the case of polygonal domains. On the contrary, the assumption on the crack path $\Gamma$ is very strong and is motivated only by technical problems in the proofs.

Let us fix a (possibly empty) Borel subset $\partial_{D} \Omega$ of $\partial \Omega$, where we will prescribe a timedependent displacement boundary condition. On the complement $\partial_{N} \Omega:=\partial \Omega \backslash \partial_{D} \Omega$ we will prescribe the traction free boundary condition.

Let $\gamma:[a, b] \rightarrow \bar{\Omega}$ be an arc-length parametrization of $\Gamma$ with $a<0<b$ and $\gamma(a), \gamma(b) \in$ $\partial \Omega$. The initial crack corresponds to $s=0$. For every $s \in[a, b]$ we set $\Gamma_{s}=\gamma([a, s])$, $\Omega_{s}:=\Omega \backslash \Gamma_{s}$, and $H_{D}^{1}\left(\Omega_{s}\right)=\left\{u \in H^{1}\left(\Omega_{s}\right): u=0 \mathcal{H}^{1}\right.$-a.e. on $\left.\partial_{D} \Omega\right\}$, where $\mathcal{H}^{1}$ denotes the one-dimensional Hausdorff measure and the values of $u$ on $\partial_{D} \Omega$ are defined using the trace operator from $H^{1}\left(\Omega_{s}\right)$ to $L^{2}(\partial \Omega)$.

Given a function $u \in H^{1}\left(\Omega_{s}\right)$ for some $s \in[a, b]$, it is convenient to regard its gradient $\nabla u$ as an element of $L^{2}\left(\Omega ; \mathbb{R}^{2}\right)$, by extending it to 0 on $\Gamma_{s}$. To underline the fact that this extension is not the distributional gradient of any extension of $u$, we shall denote it by $\widehat{\nabla} u$.

Let us fix $T>0$. The body forces $f$ satisfy

$$
f \in L^{2}\left((0, T) ; L^{2}(\Omega)\right)
$$

The Dirichlet boundary condition is prescribed using a function $w$, which, in order to use the results of [7], needs the regularity assumptions

$$
w \in L^{2}\left((0, T) ; H^{2}\left(\Omega_{0}\right)\right) \cap H^{1}\left((0, T) ; H^{1}\left(\Omega_{0}\right)\right) \cap H^{2}\left((0, T) ; L^{2}\left(\Omega_{0}\right)\right),
$$

where $\Omega_{0}:=\Omega \backslash \Gamma_{0}$ is the cracked domain defined above corresponding to $s=0$. The initial conditions $u^{0}$ and $u^{1}$ for the displacement and for its velocity satisfy

$$
u^{0}-w(0) \in H_{D}^{1}\left(\Omega_{0}\right) \text { and } u^{1} \in L^{2}(\Omega)
$$

In order to use the results of [7], throughout the paper we fix two parameters $\delta, M$ with $0<\delta<1$ and $M>0$. The uniform regularity assumptions on the length of the cracks are prescribed in the following definition. 
Definition 2.1. For every interval $\left[t_{1}, t_{2}\right] \subset[0, T]$, the class $\mathcal{C}_{\delta, M}\left(\left[t_{1}, t_{2}\right]\right)$ is composed of all functions satisfying the following conditions:

$$
\begin{gathered}
s \in C^{1,1}\left(\left[t_{1}, t_{2}\right] ;[0, b]\right) \\
0 \leq \dot{s}(t) \leq 1-\delta, \text { for every } t \in\left[t_{1}, t_{2}\right] \\
|\ddot{s}(t)| \leq M \text { for a.e. } t \in\left(t_{1}, t_{2}\right) \\
\sup _{\phi \in C_{c}^{1}\left(\left(t_{1}, t_{2}\right)\right),\|\phi\|_{\infty}<1} \int_{t_{1}}^{t_{2}} \ddot{s}(t) \dot{\phi}(t) d t \leq M .
\end{gathered}
$$

Inequality (2.7) is equivalent to saying that the third derivative of $s$ in the sense of distributions is a bounded Radon measure on $\left(t_{1}, t_{2}\right)$ and that its total variation on $\left(t_{1}, t_{2}\right)$ is bounded by $M$. Although these hypotheses have no mechanical motivation, they are needed in order to apply the existence, uniqueness, and continuous dependence results of [7].

To simplify the exposition, it is not restrictive to normalize all material parameters, so that the speed of sound is equal to one. In particular, (2.5) says that the speed of the crack tip is always less than the speed of sound.

Given a function $s \in \mathcal{C}_{\delta, M}\left(\left[t_{1}, t_{2}\right]\right)$, we consider the corresponding displacement $u$, that satisfies the wave equation

$$
\ddot{u}(t, x)-\Delta u(t, x)=f(t, x) \quad \text { for } t \in\left(t_{1}, t_{2}\right) \text { and } x \in \Omega_{s(t)},
$$

with Dirichlet boundary condition on $\partial_{D} \Omega$

$$
u(t, x)=w(t, x) \quad \text { for } t \in\left(t_{1}, t_{2}\right) \text { and } x \in \partial_{D} \Omega,
$$

Neumann boundary condition

$$
\partial_{\nu} u(t, x)=0 \quad \text { for } t \in\left(t_{1}, t_{2}\right) \text { and } x \in \partial_{N} \Omega \cup \Gamma_{s(t)},
$$

and initial conditions

$$
u\left(t_{1}, x\right)=u^{0}(x) \quad \text { and } \quad \dot{u}\left(t_{1}, x\right)=u^{1}(x) \quad \text { for } x \in \Omega_{s\left(t_{1}\right)} .
$$

In this paper we consider only the traction free boundary condition (2.10), because the corresponding weak solution has been studied in detail in [7]. The case of a nonvanishing Neumann boundary condition can be obtained under suitable regularity assumptions on the data.

In the following definition we introduce the notion of weak solution of this problem. The unusual choice of the function spaces is due to the time dependence of the cracked domain $\Omega_{s(t)}$.

Definition 2.2. Assume that $f, w, u^{0}, u^{1}$ satisfy $(2.1)-(2.3)$ and let $s \in \mathcal{C}_{\delta, M}\left(\left[t_{1}, t_{2}\right]\right)$. We say that $u$ is a weak solution of the wave equation (2.8) with boundary and initial conditions (2.9)-(2.11) on the time-dependent cracking domains $t \mapsto \Omega_{s(t)}$, if

$$
\begin{gathered}
u \in C^{1}\left(\left[t_{1}, t_{2}\right] ; L^{2}(\Omega)\right) \\
u(t)-w(t) \in H_{D}^{1}\left(\Omega_{s(t)}\right) \quad \text { for every } t \in\left[t_{1}, t_{2}\right] \\
\widehat{\nabla} u \in C^{0}\left(\left[t_{1}, t_{2}\right] ; L^{2}\left(\Omega ; \mathbb{R}^{2}\right)\right) \\
\dot{u} \in A C\left(\left[t, t_{2}\right] ; H_{D}^{-1}\left(\Omega_{s(t)}\right)\right) \text { for every } t \in\left[t_{1}, t_{2}\right) \\
\frac{1}{h}(\dot{u}(t+h)-\dot{u}(t)) \rightarrow \ddot{u}(t) \text { weakly in } H_{D}^{-1}\left(\Omega_{s(t)}\right), \text { as } h \rightarrow 0, \text { for a.e. } t \in\left(t_{1}, t_{2}\right) \\
t \mapsto\|\ddot{u}(t)\|_{H_{D}^{-1}\left(\Omega_{s(t)}\right)} \text { is integrable on }\left(t_{1}, t_{2}\right), \\
u\left(t_{1}\right)=u^{0} \text { and } \dot{u}\left(t_{1}\right)=u^{1} \text { in } L^{2}(\Omega),
\end{gathered}
$$

and for a.e. $t \in\left(t_{1}, t_{2}\right)$

$$
\langle\ddot{u}(t), \varphi\rangle+\langle\widehat{\nabla} u(t), \widehat{\nabla} \varphi\rangle=\langle f(t), \varphi\rangle \quad \text { for every } \varphi \in H_{D}^{1}\left(\Omega_{s(t)}\right) .
$$


Here and in the rest of the paper $\langle\cdot, \cdot\rangle$ denotes a duality product between spaces that are clear from the context. For instance, its first occurrence in (2.19) refers to the duality between $H_{D}^{-1}\left(\Omega_{s(t)}\right)$ and $H_{D}^{1}\left(\Omega_{s(t)}\right)$, its second occurrence refers to the duality between $L^{2}\left(\Omega ; \mathbb{R}^{2}\right)$ and $L^{2}\left(\Omega ; \mathbb{R}^{2}\right)$, and its third occurrence refers to the duality between $L^{2}(\Omega)$ and $L^{2}(\Omega)$.

Definition 2.2 is a more regular version of the definition introduced in [6]. The technical conditions (2.6) and (2.7) have been introduced in order to apply the following results of [7] on existence, uniqueness, and continuous dependence of the solutions of problem (2.8)-(2.11).

Theorem 2.3. Under the assumptions of Definition 2.2 there exists a unique weak solution of problem (2.8)-(2.11) on the time-dependent cracking domains $t \mapsto \Omega_{s(t)}$.

Proof. See [7, Example 1.14 and Corollary 2.3].

Theorem 2.4. Assume $s_{k} \in \mathcal{C}_{\delta, M}\left(\left[t_{1}, t_{2}\right]\right)$ converges uniformly to some $s \in \mathcal{C}_{\delta, M}\left(\left[t_{1}, t_{2}\right]\right)$. For every $k$, let $u_{k}$ be the weak solution of problem (2.8)-(2.11) on the cracking domain $t \mapsto \Omega_{s_{k}(t)}$ and let $u$ be the weak solution of problem (2.8)-(2.11) on the cracking domain $t \mapsto \Omega_{s(t)}$. Then

$$
\begin{gathered}
u_{k}(t, \cdot) \rightarrow u(t, \cdot) \text { strongly in } L^{2}(\Omega), \\
\widehat{\nabla} u_{k}(t, \cdot) \rightarrow \widehat{\nabla} u(t, \cdot) \text { strongly in } L^{2}\left(\Omega ; \mathbb{R}^{2}\right), \\
\dot{u}_{k}(t, \cdot) \rightarrow \dot{u}(t, \cdot) \text { strongly in } L^{2}(\Omega),
\end{gathered}
$$

for every $t \in\left[t_{1}, t_{2}\right]$.

Proof. See [7, Theorem 3.1 and Example 3.3].

Besides the class $\mathcal{C}_{\delta, M}([0, T])$, we can consider the class $\mathcal{C}_{\delta, M}^{\text {piec }}([0, T])$ defined in the following way: $s \in \mathcal{C}_{\delta, M}^{\text {piec }}([0, T])$ if and only if $s \in C^{0}([0, T])$ and there exists a finite subdivision $0=T_{0}<T_{1}<\cdots<T_{k}=T$ such that

$$
\left.s\right|_{\left[T_{j-1}, T_{j}\right]} \in \mathcal{C}_{\delta, M}\left(\left[T_{j-1}, T_{j}\right]\right) \text { for every } j=1, \ldots, k .
$$

If $f, w, u^{0}, u^{1}$ satisfy $(2.1)-(2.3)$ and $s \in \mathcal{C}_{\delta, M}^{\text {piec }}([0, T])$, then Definition 2.2 still provides a notion of weak solution of the wave equation (2.8) with boundary and initial conditions (2.9)(2.11) on the time-dependent cracking domains $t \mapsto \Omega_{s(t)}$. The existence and uniqueness of such a solution is a direct consequence of Theorem 2.3, applied to each interval $\left[T_{j-1}, T_{j}\right]$ of the subdivision.

\section{ENERGY BALANCE AND MAXIMAL DISSIPATION}

We start by discussing the issue of the energy balance for weak solutions of (2.8) with $f, w, u^{0}, u^{1}$ satisfying $(2.1)-(2.3)$ and with a general $s \in \mathcal{C}_{\delta, M}^{\text {piec }}([0, T])$. The sum of the elastic and kinetic energies of a solution $u$ at time $t$ is given by $\mathcal{E}(\widehat{\nabla} u(t), \dot{u}(t))$, where

$$
\mathcal{E}(\Psi, v):=\frac{1}{2}\|\Psi\|^{2}+\frac{1}{2}\|v\|^{2}
$$

for every $\Psi \in L^{2}\left(\Omega ; \mathbb{R}^{2}\right)$ and every $v \in L^{2}(\Omega)$. Here and in the rest of the paper $\|\cdot\|$ refers to the $L^{2}$ norm on sets that are clear from the context.

The work of the external forces on the solution $u$ over a time interval $\left[t_{1}, t_{2}\right] \subset[0, T]$ is given by

$$
\mathcal{W}_{\text {load }}\left(u ; t_{1}, t_{2}\right):=\int_{t_{1}}^{t_{2}}\langle f(t), \dot{u}(t)\rangle d t
$$


which is well defined by (2.1) and (2.12). One would expect that the work on the solution $u$ due to the varying boundary conditions $w$ over a time interval $\left[t_{1}, t_{2}\right] \subset[0, T]$ is given by

$$
\mathcal{W}_{b d r y}\left(u ; t_{1}, t_{2}\right)=\int_{t_{1}}^{t_{2}}\left\langle\partial_{\nu} u(t), \dot{w}(t)\right\rangle_{\partial_{D} \Omega} d t
$$

where $\langle\cdot, \cdot\rangle_{\partial_{D} \Omega}$ is the duality pairing between $L^{2}\left(\partial_{D} \Omega\right)$ and $L^{2}\left(\partial_{D} \Omega\right)$. Since $\dot{w}(t) \in H^{1}\left(\Omega_{0}\right)$ by $(2.2)$, its trace on $\partial_{D} \Omega$ belongs to $L^{2}\left(\partial_{D} \Omega\right)$.

Unfortunately, under the assumptions (2.12)-(2.19) the trace of the normal derivative $\partial_{\nu} u(t)$ cannot be defined, not even in a weaker sense, because $\Delta u(t)$, in general, does not belong to $L^{2}\left(\Omega_{s(t)}\right)$. This difficulty is solved by the following result.

Proposition 3.1. Assume that there exists an open neighborhood $U$ of the closure of $\partial_{D} \Omega$ such that $u \in L^{2}\left(\left(t_{1}, t_{2}\right) ; H^{2}(U \cap \Omega \backslash \Gamma)\right) \cap H^{2}\left(\left(t_{1}, t_{2}\right) ; L^{2}(U \cap \Omega)\right)$. Then $\partial_{\nu} u \in$ $L^{2}\left(\left(t_{1}, t_{2}\right) ; L^{2}\left(\partial_{D} \Omega\right)\right)$ and

$$
\begin{gathered}
\mathcal{W}_{b d r y}\left(u ; t_{1}, t_{2}\right)=\left\langle\dot{u}\left(t_{2}\right), \dot{w}\left(t_{2}\right)\right\rangle-\left\langle\dot{u}\left(t_{1}\right), \dot{w}\left(t_{1}\right)\right\rangle \\
-\int_{t_{1}}^{t_{2}}\langle\ddot{w}(t), \dot{u}(t)\rangle d t-\int_{t_{1}}^{t_{2}}\langle f(t), \dot{w}(t)\rangle d t+\int_{t_{1}}^{t_{2}}\langle\widehat{\nabla} u(t), \nabla \dot{w}(t)\rangle d t .
\end{gathered}
$$

Since the right-hand side of (3.4) is well defined under the weaker assumptions (2.12)(2.18), when $u$ is an arbitrary weak solution of (2.8) we consider (3.4) as the weak definition of the work $\mathcal{W}_{b d r y}\left(u ; t_{1}, t_{2}\right)$ due to the varying boundary conditions $w$.

Proof of Proposition 3.1. Since $u \in L^{2}\left(\left(t_{1}, t_{2}\right) ; H^{2}\left(U \cap \Omega^{ \pm}\right)\right.$, and $U$ is a neighborhood of $\partial_{D} \Omega$, by the continuity of the trace operator we have $\partial_{\nu} u \in L^{2}\left(\left(t_{1}, t_{2}\right) ; L^{2}\left(\partial_{D} \Omega\right)\right)$. Moreover our assumptions, together with (2.19), imply that

$$
\ddot{u}(t)-\Delta u(t)=f(t) \quad \text { in } L^{2}(U \cap \Omega \backslash \Gamma)
$$

for a.e. $t \in\left(t_{1}, t_{2}\right)$. Let us fix $\varphi \in C_{c}^{1}\left(\mathbb{R}^{2}\right)$ with $\operatorname{supp} \varphi \subset U$ and $\varphi=1$ on $\partial_{D} \Omega$. Integrating by parts with respect to space, using (3.5) and the equality $\partial_{\nu} u=0$ on $\partial_{N} \Omega \cup \Gamma_{s(t)}$, we obtain

$$
\begin{gathered}
\left\langle\partial_{\nu} u(t), \dot{w}(t)\right\rangle_{\partial_{D} \Omega}=\left\langle\partial_{\nu} u(t), \varphi \dot{w}(t)\right\rangle_{\partial_{D} \Omega}=\langle\Delta u(t), \varphi \dot{w}(t)\rangle+\langle\widehat{\nabla} u(t), \nabla(\varphi \dot{w}(t))\rangle \\
=\langle\ddot{u}(t), \varphi \dot{w}(t)\rangle-\langle f(t), \varphi \dot{w}(t)\rangle+\langle\widehat{\nabla} u(t), \nabla(\varphi \dot{w}(t))\rangle .
\end{gathered}
$$

Since $(1-\varphi) \dot{w}(t) \in H_{D}^{1}\left(\Omega_{s(t)}\right)$ for a.e. $t \in(0, T)$, from (2.19) we get

$$
\langle\ddot{u}(t),(1-\varphi) \dot{w}(t)\rangle+\langle\widehat{\nabla} u(t), \nabla((1-\varphi) \dot{w}(t))\rangle-\langle f(t),(1-\varphi) \dot{w}(t)\rangle=0
$$

for a.e. $t \in(0, T)$. Adding the left-hand side of (3.7) to the right-hand side of (3.6), from (3.3) we deduce that

$$
\mathcal{W}_{b d r y}\left(u ; t_{1}, t_{2}\right)=\int_{t_{1}}^{t_{2}}\langle\ddot{u}(t), \dot{w}(t)\rangle d t-\int_{t_{1}}^{t_{2}}\langle f(t), \dot{w}(t)\rangle d t+\int_{t_{1}}^{t_{2}}\langle\widehat{\nabla} u(t), \nabla \dot{w}(t)\rangle d t
$$

Integrating by parts with respect to time we finally obtain (3.4).

The total work on the solution $u$ over a time interval $\left[t_{1}, t_{2}\right] \subset[0, T]$ is defined by

$$
\mathcal{W}\left(u ; t_{1}, t_{2}\right):=\mathcal{W}_{\text {load }}\left(u ; t_{1}, t_{2}\right)+\mathcal{W}_{\text {bdry }}\left(u ; t_{1}, t_{2}\right),
$$

where $\mathcal{W}_{\text {load }}$ is defined by $(3.2)$ and $\mathcal{W}_{\text {bdry }}$ is defined by (3.4).

Remark 3.2. If $s(\cdot)$ is constant in the interval $\left[t_{1}, t_{2}\right] \subset[0, T]$, then every solution $u$ of the wave equation (2.8) according to Definition 2.2, satisfies the energy equality

$$
\mathcal{E}\left(\widehat{\nabla} u\left(t_{2}\right), \dot{u}\left(t_{2}\right)\right)-\mathcal{E}\left(\widehat{\nabla} u\left(t_{1}\right), \dot{u}\left(t_{1}\right)\right)=\mathcal{W}\left(u ; t_{1}, t_{2}\right) .
$$

This result is well known for the wave equation in a sufficiently smooth domain. For an arbitrary bounded open set one can follow the lines of the proof of $[8$, Lemma 1]. An 
alternative way is to perturb the wave equation with a small damping term and use the fact that the energy balance always holds for the damped wave equation (see [6, Theorem 3.2]).

We now introduce the class of admissible functions $s$ which satisfy the dynamic energydissipation balance.

Definition 3.3. Assume that $f, w, u^{0}, u^{1}$ satisfy (2.1)-(2.3) and let $s^{0} \in[0, b]$. The class $\mathcal{S}$ is composed of all functions $s \in \mathcal{C}_{\delta, M}^{\text {piec }}([0, T])$, with $s(0)=s^{0}$, such that the unique weak solution $u$ of (2.8)-(2.11) on the time-dependent cracking domains $t \mapsto \Omega_{s(t)}$ satisfies the dynamic energy-dissipation balance

$$
\left.\mathcal{E}\left(\widehat{\nabla} u\left(t_{2}\right), \dot{u}\left(t_{2}\right)\right)-\mathcal{E}\left(\widehat{\nabla} u\left(t_{1}\right)\right), \dot{u}\left(t_{1}\right)\right)+s\left(t_{2}\right)-s\left(t_{1}\right)=\mathcal{W}\left(u ; t_{1}, t_{2}\right)
$$

for every interval $\left[t_{1}, t_{2}\right] \subset[0, T]$.

The meaning of (3.8) is conservation of energy: The work done on the system is balanced by the change in mechanical energy $\left.\mathcal{E}\left(\widehat{\nabla} u\left(t_{2}\right), \dot{u}\left(t_{2}\right)\right)-\mathcal{E}\left(\widehat{\nabla} u\left(t_{1}\right)\right), \dot{u}\left(t_{1}\right)\right)$ and by the energy dissipated in the process of crack production in the same time interval $\left[t_{1}, t_{2}\right]$, which is assumed to be given by $s\left(t_{2}\right)-s\left(t_{1}\right)$, consistent with the Griffith theory.

Remark 3.4. Note that the class $\mathcal{S}$ is not empty: by Remark 3.2 it contains at least the constant function $s(t)=s^{0}$ for all $t \in[0, T]$. We shall see in Section 4 that there are examples where $\mathcal{S}$ contains nonconstant functions.

To define the notion of $\eta$-maximal dissipation solution of the dynamic crack evolution, for every $s \in \mathcal{S}$ and $\tau \in[0, T]$ we introduce the class $\mathcal{A}(s, \tau)$ of admissible comparison functions, defined as the class of functions $\sigma \in \mathcal{S}$, with $\sigma(t) \geq s(t)$ for all $t \in[0, \tau]$, such that $\dot{\sigma}$ is continuous where $\dot{s}$ is continuous.

Definition 3.5. Assume that $f, w, u^{0}, u^{1}$ satisfy (2.1)-(2.3) and let $s^{0} \in[0, b]$ and $\eta>0$. We say that $s$ is an $\eta$-maximal dissipation solution of the dynamic crack evolution problem corresponding to these data if $s \in \mathcal{S}$ and for every $\tau \in[0, T]$ there is no $\sigma \in \mathcal{A}(s, \tau)$ such that $\sigma(\tau)>s(\tau)+\eta$

Theorem 3.6. Assume that $f, w, u^{0}, u^{1}$ satisfy (2.1)-(2.3) and let $s^{0} \in[0, b]$ and $\eta>0$. Then there exists an $\eta$-maximal dissipation solution of the dynamic crack evolution problem corresponding to these data.

The main difficulty in the definition of an $\eta$-maximal dissipation solution is the variability of $\tau$. To overcome this problem we discretize time and in each time interval we prove the existence of a maximal function $s$ among all functions satisfying our regularity requirements and the energy equality. Then we prove that the function obtained by glueing together these maximal functions is an $\eta$-maximal dissipation solution, provided that the length of each time interval is less that $\eta$ (recall that the speed of sound is normalized to one).

Proof of Theorem 3.6. Let us fix a finite subdivision $0=T_{0}<T_{1}<\cdots<T_{k}=T$ such that $T_{j}-T_{j-1} \leq \eta$ for every $j=1, \ldots, k$. The solution will be constructed recursively in the intervals $\left[T_{j-1}, T_{j}\right]$. Let $\mathcal{S}_{1}$ be the set of all functions $s \in \mathcal{C}_{\delta, M}\left(\left[T_{0}, T_{1}\right]\right)$, with $s\left(T_{0}\right)=s^{0}$, such that the unique weak solution $u$ of (2.8)-(2.11) on the time-dependent cracking domains $t \mapsto \Omega_{s(t)}, t \in\left[T_{0}, T_{1}\right]$, satisfies the dynamic energy-dissipation balance (3.8) for every interval $\left[t_{1}, t_{2}\right] \subset\left[T_{0}, T_{1}\right]$. In Lemma 3.7 below we shall prove that there exists $s_{1} \in \mathcal{S}_{1}$ such that

$$
\int_{T_{0}}^{T_{1}} s_{1}(t) d t=\max _{s \in \mathcal{S}_{1}} \int_{T_{0}}^{T_{1}} s(t) d t .
$$

Finally, we define $u_{1}$ to be the unique weak solution $u$ of (2.8)-(2.11) on the time-dependent cracking domains $t \mapsto \Omega_{s_{1}(t)}, t \in\left[T_{0}, T_{1}\right]$. 
Suppose now that $s_{j} \in \mathcal{C}_{\delta, M}\left(\left[T_{j-1}, T_{j}\right]\right)$ and $u_{j} \in C^{1}\left(\left[T_{j-1}, T_{j}\right] ; L^{2}(\Omega)\right)$ are defined for some $1 \leq j<k$. Then we define $\mathcal{S}_{j+1}$ as the set of all functions $s \in \mathcal{C}_{\delta, M}\left(\left[T_{j}, T_{j+1}\right]\right)$, with $s\left(T_{j}\right)=s_{j}\left(T_{j}\right)$, such that the unique weak solution $u$ of (2.8)-(2.11), with $u^{0}$ and $u^{1}$ replaced by $u_{j}\left(T_{j}\right)$ and $\dot{u}_{j}\left(T_{j}\right)$, on the time-dependent cracking domains $t \mapsto \Omega_{s(t)}, t \in$ $\left[T_{j}, T_{j+1}\right]$, satisfies the dynamic energy-dissipation balance $(3.8)$ for every interval $\left[t_{1}, t_{2}\right] \subset$ $\left[T_{j}, T_{j+1}\right]$. As above, there exists $s_{j+1} \in \mathcal{S}_{j+1}$ such that

$$
\int_{T_{j}}^{T_{j+1}} s_{j+1}(t) d t=\max _{s \in \mathcal{S}_{j+1}} \int_{T_{j}}^{T_{j+1}} s(t) d t .
$$

Finally, we define $u_{j+1}$ to be the unique weak solution $u$ of (2.8)-(2.11), with $u^{0}$ and $u^{1}$ replaced by $u_{j}\left(T_{j}\right)$ and $\dot{u}_{j}\left(T_{j}\right)$, on the time-dependent cracking domains $t \mapsto \Omega_{s_{j+1}(t)}$, $t \in\left[T_{j}, T_{j+1}\right]$.

After $k$ steps we have constructed $s_{j}$ and $u_{j}$ on each interval $\left[T_{j-1}, T_{j}\right], j=1, \ldots, k$, in such a way that $s_{j}\left(T_{j}\right)=s_{j+1}\left(T_{j}\right), u_{j}\left(T_{j}\right)=u_{j+1}\left(T_{j}\right)$, and $\dot{u}_{j}\left(T_{j}\right)=\dot{u}_{j+1}\left(T_{j}\right)$ for $j=1, \ldots, k-1$. Moreover $s_{1}(0)=s^{0}, u_{1}(0)=u^{0}$, and $\dot{u}_{1}(0)=u^{1}$. We now set $s(t):=s_{j}(t)$ and $u(t):=u_{j}(t)$ for $t \in\left[T_{j-1}, T_{j}\right], j=1, \ldots, k$. The previous remark shows that $s \in \mathcal{S}$ and that $u$ is the unique weak solution of (2.8)-(2.11) on the time-dependent cracking domains $t \mapsto \Omega_{s(t)}, t \in[0, T]$.

To show that $s$ satisfies the maximality property in Definition 3.5, we assume by contradiction that there exist $\tau \in[0, T]$ and $\sigma \in \mathcal{A}(s, \tau)$ such that

$$
\sigma(\tau)>s(\tau)+\eta
$$

Let $\tau_{0}:=\inf \{t \in[0, \tau]: \sigma(t)>s(t)\}$ and let $j \in\{0, \ldots, k-1\}$ be such that $\tau_{0} \in\left[T_{j}, T_{j+1}\right)$. We claim that $T_{j+1} \leq \tau$. If not, the inequality $\dot{\sigma}(t) \leq 1-\delta$, would imply $\sigma(\tau)-\sigma\left(\tau_{0}\right) \leq$ $(1-\delta)\left(T_{j+1}-T_{j}\right)<\eta$, hence $\sigma(\tau)<\sigma\left(\tau_{0}\right)+\eta=s\left(\tau_{0}\right)+\eta \leq s(\tau)+\eta$, which contradicts (3.10). Therefore $T_{j+1} \leq \tau$.

By the definition of $\mathcal{A}(s, \tau)$ given before Definition 3.5 we have $\sigma \in \mathcal{C}_{\delta, M}\left(\left[T_{j}, T_{j+1}\right]\right)$. Since $\sigma(t)=s(t)$ for $t \in\left[0, \tau_{0}\right]$, by the uniqueness of the solution to the wave equation we deduce that $u_{\sigma}\left(T_{j}\right)=u\left(T_{j}\right)=u_{j}\left(T_{j}\right)$ and $\dot{u}_{\sigma}\left(T_{j}\right)=\dot{u}\left(T_{j}\right)=\dot{u}_{j}\left(T_{j}\right)$, where $u_{\sigma}$ and $u$ denote the unique solutions to the wave equation on $[0, T]$ in the cracking domains corresponding to $\sigma$ and $s$, respectively. This implies that $\left.\sigma\right|_{\left[T_{j}, T_{j+1}\right]} \in \mathcal{S}_{j+1}$, hence (3.9) gives

$$
\int_{T_{j}}^{T_{j+1}} s(t) d t=\int_{T_{j}}^{T_{j+1}} s_{j+1}(t) d t \geq \int_{T_{j}}^{T_{j+1}} \sigma(t) d t .
$$

Since $\sigma(t) \geq s(t)$ for $t \in[0, \tau]$ and $T_{j+1} \leq \tau$, we have $\sigma(t) \geq s(t)$ for every $t \in\left[T_{j}, T_{j+1}\right]$. As both functions are continuous in $\left[T_{j}, T_{j+1}\right]$, we conclude that $\sigma(t)=s(t)$ for every $t \in\left[T_{j}, T_{j+1}\right]$, which contradicts the definition of $\tau_{0}$ and the choice of $j$.

To conclude the proof of Theorem 3.6 we need the following lemma.

Lemma 3.7. For every $j=1, \ldots, k$ there exists $s_{j} \in \mathcal{S}_{j}$ such that

$$
\int_{T_{j-1}}^{T_{j}} s_{j}(t) d t=\max _{s \in \mathcal{S}_{j}} \int_{T_{j-1}}^{T_{j}} s(t) d t
$$

Proof. Fix $j=1, \ldots, k$ and set $I_{\max }:=\sup _{s \in \mathcal{S}_{j}} \int_{T_{j-1}}^{T_{j}} s(t) d t$ and, for every $n \in \mathbb{N}$, let $s_{n} \in \mathcal{S}_{j}$ be such that

$$
\int_{0}^{T} s_{n}(t) d t \geq I_{\max }-\frac{1}{n} .
$$

By the compactness of $\mathcal{C}_{\delta, M}\left(\left[T_{j-1}, T_{j}\right]\right)$ there exists a subsequence of $s_{n}$, not relabeled, and a function $s \in \mathcal{C}_{\delta, M}\left(\left[T_{j-1}, T_{j}\right]\right)$ such that $s_{n} \rightarrow s$ in $C^{1}\left(\left[T_{j-1}, T_{j}\right]\right)$. 
Since $s_{n} \in \mathcal{S}_{j}$, the weak solutions $u_{n}$ to the wave equation corresponding to $s_{n}$ and to the data $f, w, u_{j-1}\left(T_{j-1}\right)$, and $\dot{u}_{j-1}\left(T_{j-1}\right)\left(u^{0}\right.$ and $u^{1}$ if $\left.j=1\right)$, satisfy the energy equality

$$
\mathcal{E}\left(\widehat{\nabla} u_{n}\left(t_{2}\right), \dot{u}_{n}\left(t_{2}\right)\right)-\mathcal{E}\left(\widehat{\nabla} u_{n}\left(t_{1}\right), \dot{u}_{n}\left(t_{1}\right)\right)+s_{n}\left(t_{2}\right)-s_{n}\left(t_{1}\right)=\mathcal{W}\left(u_{n} ; t_{1}, t_{2}\right)
$$

for every interval $\left[t_{1}, t_{2}\right] \subset\left[T_{j-1}, T_{j}\right]$. Let $u$ be the solution to the wave equation corresponding to $s$ and to the same data $f, w, u^{0}, u^{1}$. Passing to the limit thanks to Theorem 2.4, by (2.20)-(2.22), we obtain (3.8), so that $s \in \mathcal{S}_{j}$. On the other hand, from (3.12) we get $\int_{0}^{T} s(t) d t=I_{\max }$, which gives $(3.11)$.

\section{An Example}

The results of the previous section provide the existence of an $\eta$-maximal dissipation solution. However, if the elastic singularity of the initial data is not large enough, the dynamic energy-dissipation balance is satisfied only if the crack does not grow, and consequently the $\eta$-maximal dissipation solution is given by the stationary crack.

The example presented in this section gives a complete mathematical proof of the fact that, in some cases, it is possible to grow the crack while balancing energy. In particular, this implies that a stationary crack does not always satisfy $\eta$-maximal dissipation, and therefore it does not always satisfy maximal dissipation in the sense considered in the Introduction.

In this example $0 \in \Omega, \partial_{D} \Omega=\partial \Omega, \Gamma$ is the intersection of $\bar{\Omega}$ with the $x_{1}$-axis, and the parametrization is given by $\gamma(s)=(s, 0) \in \Omega$ for every $s \in(a, b)$, so that $\Omega_{s}=\Omega \backslash\left\{\left(x_{1}, 0\right)\right.$ : $\left.x_{1} \leq s\right\}$. Finally, we assume that there are no body forces, so $f=0$.

We will construct time-dependent Dirichlet boundary conditions $w(t)$ in such a way that a crack growing with constant speed satisfies the dynamic energy-dissipation balance.

To this aim, we first fix the speed $c$, with $0<c<1$ and $c T<b$, and we consider the classical singularity of the solution of the wave equation at a crack tip moving with constant speed. This is expressed by means of the function $\psi: \mathbb{R}^{2} \backslash\left\{\left(x_{1}, 0\right): x_{1} \leq 0\right\} \rightarrow \mathbb{R}$ defined by

$$
\psi(x):=\mathcal{I} m\left(\sqrt{x_{1}+i x_{2}}\right)=\sqrt{\rho} \sin (\theta / 2),
$$

where $\mathcal{I} m$ denotes the imaginary part and $\rho, \theta$ are the polar coordinates of $x=\left(x_{1}, x_{2}\right)$, with $-\pi<\theta<\pi$. Since $\Delta \psi=0$ in $\mathbb{R}^{2} \backslash\left\{\left(x_{1}, 0\right): x_{1} \leq 0\right\}$, and $\partial_{\nu} \psi=0$ on $\left\{\left(x_{1}, 0\right): x_{1}<\right.$ $0\}$, it is easy to check that for every $\kappa \in \mathbb{R}$ the function

$$
u_{\kappa}(t, x):=\kappa \psi\left(\frac{x_{1}-c t}{\sqrt{1-c^{2}}}, x_{2}\right),
$$

defined for $t \in[0, T]$ and $x \in \Omega_{c t}$, is a singular solution of the wave equation (2.8) satisfying the homogeneous Neumann boundary condition (2.10) on the crack, where $s(t):=c t$. Since $\psi \in H^{1}\left(B \backslash\left\{\left(x_{1}, 0\right): x_{1} \leq 0\right\}\right)$ for every bounded open set $B \subset \mathbb{R}^{2}$, we conclude that $u_{\kappa}$ also satisfies the regularity assumptions (2.12), (2.14), (2.15), (2.16), (2.17), and the corresponding weak formulation (2.19) of the wave equation.

We now fix the initial conditions $u_{\kappa}^{0}$ and $u_{\kappa}^{1}$, and the Dirichlet boundary condition $w_{\kappa}$ in such a way that $u_{\kappa}$ is the unique solution of the wave equation in the time-dependent cracking domain $t \mapsto \Omega_{c t}$. We define $u_{\kappa}^{0}:=u_{\kappa}(0)$ and $u_{\kappa}^{1}:=\dot{u}_{\kappa}(0)$. According to (2.2), the function $w_{\kappa}(t)$ used to prescribe the Dirichlet boundary condition must be regular on the domain $\Omega_{0}$ corresponding to $t=0$.

To ensure this regularity we choose an open neighborhood $U$ of $\partial \Omega$, such that $\Omega \cap(U \backslash \Gamma) \subset$ $\Omega_{s}$ for every $s \in[0, c T]$, and a function $\varphi \in C_{c}^{\infty}\left(\mathbb{R}^{2}\right)$, with $\operatorname{supp} \varphi \subset U$ and $\varphi=1$ on $\partial \Omega$. Then the function

$$
w_{\kappa}(t, x):=\varphi(x) u_{\kappa}(t, x)
$$

belongs to $C^{\infty}\left([0, T] \times \Omega_{0}\right)$ and $u_{\kappa}$ satisfies the Dirichlet boundary condition (2.13). 
We want to determine $\kappa$ so that the dynamic energy-dissipation balance (3.8) be satisfied for every interval $\left[t_{1}, t_{2}\right] \subset[0, T]$. Taking into account Proposition 3.1, the dynamic energydissipation balance becomes

$$
\frac{1}{2}\left\|\widehat{\nabla} u_{\kappa}(t)\right\|^{2}+\frac{1}{2}\left\|\dot{u}_{\kappa}(t)\right\|^{2}+c t=\frac{1}{2}\left\|\widehat{\nabla} u_{\kappa}(0)\right\|^{2}+\frac{1}{2}\left\|\dot{u}_{\kappa}(0)\right\|^{2}+\int_{0}^{t}\left\langle\partial_{\nu} u_{\kappa}(\tau), \dot{u}_{\kappa}(\tau)\right\rangle_{\partial \Omega} d \tau
$$

for every $t \in[0, T]$.

Let

$$
\Omega_{c t}:=\Omega \backslash\left\{\left(x_{1}, 0\right): x_{1} \leq c t\right\} \quad \text { and } \quad \Omega_{c t}^{\varepsilon}=\Omega_{c t} \backslash E_{c t}^{\varepsilon}
$$

where

$$
E_{c t}^{\varepsilon}=\left\{x=\left(x_{1}, x_{2}\right) \in \mathbb{R}^{2}: \frac{\left(x_{1}-c t\right)^{2}}{1-c^{2}}+x_{2}^{2} \leq \varepsilon^{2}\right\}
$$

On $\Omega_{c t}^{\varepsilon}$ we multiply both terms of equation (2.8) by $\dot{u}_{\kappa}(t)$, which is smooth in this set. Since $u_{\kappa}(t)$ satisfies the homogeneous Neumann boundary condition (2.10) on $\Gamma_{c t}$, integrating by parts we obtain

$$
\frac{1}{2} \int_{\Omega_{c t}^{\varepsilon}} \frac{d}{d t}\left|\dot{u}_{\kappa}(t)\right|^{2} d x+\frac{1}{2} \int_{\Omega_{c t}^{\varepsilon}} \frac{d}{d t}\left|\widehat{\nabla} u_{\kappa}(t)\right|^{2} d x=\int_{\partial \Omega} \partial_{\nu} u_{\kappa}(t) \dot{u}_{\kappa}(t) d \mathcal{H}^{1}-\int_{\partial E_{c t}^{\varepsilon}} \partial_{\nu} u_{\kappa}(t) \dot{u}_{\kappa}(t) d \mathcal{H}^{1},
$$

where $\nu=\left(\nu_{1}, \nu_{2}\right)$ is the outer unit normal to $\Omega$ and to $E_{c t}^{\varepsilon}$. By Reynolds transport theorem, from the previous equation we obtain

$$
\begin{aligned}
& \frac{d}{d t}\left(\frac{1}{2} \int_{\Omega_{c t}^{\varepsilon}}\left|\dot{u}_{\kappa}(t)\right|^{2} d x+\frac{1}{2} \int_{\Omega_{c t}^{\varepsilon}}\left|\widehat{\nabla} u_{\kappa}(t)\right|^{2} d x\right) \\
& =\frac{1}{2} \int_{\Omega_{c t}^{\varepsilon}} \frac{d}{d t}\left|\dot{u}_{\kappa}(t)\right|^{2} d x+\frac{1}{2} \int_{\Omega_{c t}^{\varepsilon}} \frac{d}{d t}\left|\widehat{\nabla} u_{\kappa}(t)\right|^{2} d x-\frac{c}{2} \int_{\partial E_{c t}^{\varepsilon}}\left|\dot{u}_{\kappa}(t)\right|^{2} \nu_{1} d \mathcal{H}^{1}-\frac{c}{2} \int_{\partial E_{c t}^{\varepsilon}}\left|\widehat{\nabla} u_{\kappa}(t)\right|^{2} \nu_{1} d \mathcal{H}^{1} \\
& =\int_{\partial \Omega} \partial_{\nu} u_{\kappa}(t) \dot{u}_{\kappa}(t) d \mathcal{H}^{1}-\int_{\partial E_{c t}^{\varepsilon}}\left(\partial_{\nu} u_{\kappa}(t) \dot{u}_{\kappa}(t)+\frac{c}{2}\left|\dot{u}_{\kappa}(t)\right|^{2} \nu_{1}+\frac{c}{2}\left|\widehat{\nabla} u_{\kappa}(t)\right|^{2} \nu_{1}\right) d \mathcal{H}^{1} .
\end{aligned}
$$

Integrating on $\left[t_{1}, t_{2}\right] \subset[0, T]$ we get

$$
\begin{aligned}
& \frac{1}{2} \int_{\Omega \backslash E_{c t_{2}}^{\varepsilon}}\left|\widehat{\nabla} u_{\kappa}\left(t_{2}\right)\right|^{2} d x+\frac{1}{2} \int_{\Omega \backslash E_{c t_{2}}^{\varepsilon}}\left|\dot{u}_{\kappa}\left(t_{2}\right)\right|^{2} d x-\frac{1}{2} \int_{\Omega \backslash E_{c t_{1}}^{\varepsilon}}\left|\widehat{\nabla} u_{\kappa}\left(t_{1}\right)\right|^{2} d x-\frac{1}{2} \int_{\Omega \backslash E_{c t_{1}}^{\varepsilon}}\left|\dot{u}_{\kappa}\left(t_{1}\right)\right|^{2} d x \\
& =\int_{t_{1}}^{t_{2}} \int_{\partial \Omega} \partial_{\nu} u_{\kappa}(t) \dot{u}_{\kappa}(t) d \mathcal{H}^{1} d t-\int_{t_{1}}^{t_{2}} \int_{\partial E_{c t}^{\varepsilon}}\left(\partial_{\nu} u_{\kappa}(t) \dot{u}_{\kappa}(t)+\frac{c}{2}\left|\dot{u}_{\kappa}(t)\right|^{2} \nu_{1}+\frac{c}{2}\left|\widehat{\nabla} u_{\kappa}(t)\right|^{2} \nu_{1}\right) d \mathcal{H}^{1} d t .
\end{aligned}
$$

Note that $u_{\kappa}\left(t_{2}\right)\left(x_{1}, x_{2}\right)=u_{\kappa}\left(t_{1}\right)\left(x_{1}-c\left(t_{2}-t_{1}\right), x_{2}\right)$ and $E_{c t_{2}}^{\varepsilon}$ is the corresponding translation of $E_{c t_{1}}^{\varepsilon}$, hence a change of variables gives

$$
\int_{E_{c t_{1}}^{\varepsilon}}\left(\left|\dot{u}_{\kappa}\left(t_{1}\right)\right|^{2}+\left|\widehat{\nabla} u_{\kappa}\left(t_{1}\right)\right|^{2}\right) d x=\int_{E_{c t_{2}}^{\varepsilon}}\left(\left|\dot{u}_{\kappa}\left(t_{2}\right)\right|^{2}+\left|\widehat{\nabla} u_{\kappa}\left(t_{2}\right)\right|^{2}\right) d x .
$$

Adding these terms to the previous equality we obtain

$$
\begin{aligned}
& \frac{1}{2}\left\|\widehat{\nabla} u_{\kappa}\left(t_{2}\right)\right\|^{2}+\frac{1}{2}\left\|\dot{u}_{\kappa}\left(t_{2}\right)\right\|^{2}-\frac{1}{2}\left\|\widehat{\nabla} u_{\kappa}\left(t_{1}\right)\right\|^{2}-\frac{1}{2}\left\|\dot{u}_{\kappa}\left(t_{1}\right)\right\|^{2} \\
& =\int_{t_{1}}^{t_{2}} \int_{\partial \Omega} \partial_{\nu} u_{\kappa}(t) \dot{u}_{\kappa}(t) d \mathcal{H}^{1} d t-\int_{t_{1}}^{t_{2}} \int_{\partial E_{c t}^{\varepsilon}}\left(\partial_{\nu} u_{\kappa}(t) \dot{u}_{\kappa}(t)+\frac{c}{2}\left|\dot{u}_{\kappa}(t)\right|^{2} \nu_{1}+\frac{c}{2}\left|\widehat{\nabla} u_{\kappa}(t)\right|^{2} \nu_{1}\right) d \mathcal{H}^{1} d t .
\end{aligned}
$$

Therefore, the dynamic energy-dissipation balance (4.4) is satisfied provided that

$$
c\left(t_{2}-t_{1}\right)=\int_{t_{1}}^{t_{2}} \int_{\partial E_{c t}^{\varepsilon}}\left(\partial_{\nu} u_{\kappa}(t) \dot{u}_{\kappa}(t)+\frac{c}{2}\left|\dot{u}_{\kappa}(t)\right|^{2} \nu_{1}+\frac{c}{2}\left|\widehat{\nabla} u_{\kappa}(t)\right|^{2} \nu_{1}\right) d \mathcal{H}^{1} d t
$$


Using (4.2), we express the integrands in terms of the function $\psi$ defined in (4.1), and we obtain

$$
\begin{gathered}
I:=\int_{\partial E_{c t}^{\varepsilon}}\left(\partial_{\nu} u_{\kappa}(t) \dot{u}_{\kappa}(t)+\frac{c}{2}\left|\dot{u}_{\kappa}(t)\right|^{2} \nu_{1}+\frac{c}{2}\left|\widehat{\nabla} u_{\kappa}(t)\right|^{2} \nu_{1}\right) d \mathcal{H}^{1} \\
=\kappa^{2} \int_{\partial E_{c t}^{\varepsilon}}\left[\left(\frac{1}{\sqrt{1-c^{2}}} \partial_{1} \psi\left(\frac{x_{1}-c t}{\sqrt{1-c^{2}}}, x_{2}\right) \nu_{1}+\partial_{2} \psi\left(\frac{x_{1}-c t}{\sqrt{1-c^{2}}}, x_{2}\right) \nu_{2}\right) \frac{-c}{\sqrt{1-c^{2}}} \partial_{1} \psi\left(\frac{x_{1}-c t}{\sqrt{1-c^{2}}}, x_{2}\right)\right. \\
\quad+\frac{c}{2}\left(\frac{1}{1-c^{2}}\left(\partial_{1} \psi\left(\frac{x_{1}-c t}{\sqrt{1-c^{2}}}, x_{2}\right)\right)^{2}+\left(\partial_{2} \psi\left(\frac{x_{1}-c t}{\sqrt{1-c^{2}}}, x_{2}\right)\right)^{2}\right) \nu_{1} \\
\left.\quad+\frac{c}{2} \frac{c^{2}}{1-c^{2}}\left(\partial_{1} \psi\left(\frac{x_{1}-c t}{\sqrt{1-c^{2}}}, x_{2}\right)\right)^{2} \nu_{1}\right] d \mathcal{H}^{1} \\
=c \kappa^{2} \int_{\partial E_{c t}^{\varepsilon}}\left[-\frac{1}{2}\left(\partial_{1} \psi\left(\frac{x_{1}-c t}{\sqrt{1-c^{2}}}, x_{2}\right)\right)^{2} \nu_{1}+\frac{1}{2}\left(\partial_{2} \psi\left(\frac{x_{1}-c t}{\sqrt{1-c^{2}}}, x_{2}\right)\right)^{2} \nu_{1}\right. \\
\left.+\frac{-1}{\sqrt{1-c^{2}}} \partial_{1} \psi\left(\frac{x_{1}-c t}{\sqrt{1-c^{2}}}, x_{2}\right) \partial_{2} \psi\left(\frac{x_{1}-c t}{\sqrt{1-c^{2}}}, x_{2}\right) \nu_{2}\right] d \mathcal{H}^{1}
\end{gathered}
$$

We now parametrize $\partial E_{c t}^{\varepsilon}$ in order to compute the integrals:

$$
x_{1}-c t=\varepsilon \sqrt{1-c^{2}} \cos \theta, \quad x_{2}=\varepsilon \sin \theta, \quad \theta \in(-\pi, \pi) .
$$

Then the outer unit normal and the length element are given by

$$
\nu(x)=\frac{\left(\cos \theta, \sqrt{1-c^{2}} \sin \theta\right)}{\sqrt{\left(1-c^{2}\right) \sin ^{2} \theta+\cos ^{2} \theta}} \text { and } d \mathcal{H}^{1}=\varepsilon \sqrt{\left(1-c^{2}\right) \sin ^{2} \theta+\cos ^{2} \theta} d \theta
$$

Hence

$$
\begin{gathered}
I=c \kappa^{2} \int_{-\pi}^{\pi} \varepsilon\left[-\frac{1}{2}\left(\partial_{1} \psi(\varepsilon \cos \theta, \varepsilon \sin \theta)\right)^{2} \cos \theta+\frac{1}{2}\left(\partial_{2} \psi(\varepsilon \cos \theta, \varepsilon \sin \theta)\right)^{2} \cos \theta\right. \\
\left.+\frac{-1}{\sqrt{1-c^{2}}} \partial_{1} \psi(\varepsilon \cos \theta, \varepsilon \sin \theta) \partial_{2} \psi(\varepsilon \cos \theta, \varepsilon \sin \theta) \sqrt{1-c^{2}} \sin \theta\right] d \theta \\
=\frac{c}{2} \kappa^{2} \int_{-\pi}^{\pi}\left[-\left(\partial_{1} \psi(\cos \theta, \sin \theta)\right)^{2} \cos \theta+\left(\partial_{2} \psi(\cos \theta, \sin \theta)\right)^{2} \cos \theta\right. \\
\left.-2 \partial_{1} \psi(\cos \theta, \sin \theta) \partial_{2} \psi(\cos \theta, \sin \theta) \sin \theta\right] d \theta,
\end{gathered}
$$

where we have used the fact that $\partial_{1} \psi$ and $\partial_{2} \psi$ are positively homogeneous of degree $-1 / 2$ by (4.1). Substituting in (4.5) we deduce that the dynamic energy-dissipation balance (4.4) is satisfied provided that

$$
\begin{gathered}
1=\frac{\kappa^{2}}{2} \int_{-\pi}^{\pi}\left[-\left(\partial_{1} \psi(\cos \theta, \sin \theta)\right)^{2} \cos \theta+\left(\partial_{2} \psi(\cos \theta, \sin \theta)\right)^{2} \cos \theta\right. \\
\left.-2 \partial_{1} \psi(\cos \theta, \sin \theta) \partial_{2} \psi(\cos \theta, \sin \theta) \sin \theta\right] d \theta
\end{gathered}
$$

This shows that $\kappa$ does not depend on the crack speed $c$ (nor on $t_{1}, t_{2}$, and $\varepsilon$, which can be deduced also by simpler arguments).

Using the explicit expression (4.1) of the function $\psi$ we obtain that

$$
\partial_{1} \psi(\cos \theta, \sin \theta)=-\frac{\operatorname{sign} \theta}{2 \sqrt{2}} \sqrt{1-\cos \theta} \quad \text { and } \quad \partial_{2} \psi(\cos \theta, \sin \theta)=\frac{1}{2 \sqrt{2}} \sqrt{1+\cos \theta} .
$$

Hence the dynamic energy-dissipation balance (4.4) is satisfied if

$$
1=\frac{\kappa^{2}}{2} \int_{-\pi}^{\pi}\left[-\frac{1}{8}(1-\cos \theta) \cos \theta+\frac{1}{8}(1+\cos \theta) \cos \theta+\frac{2}{8} \sin ^{2} \theta\right] d \theta=\frac{\kappa^{2}}{2} \frac{\pi}{2},
$$


and we obtain that in this case

$$
\kappa^{2}=\frac{4}{\pi}
$$

We remark that this result does not agree with formula (81) in [19]. The discrepancy can be explained by the fact that we multiply equation $(2.8)$ by $\dot{u}_{\kappa}(t)$ and integrate by parts only in $\Omega_{c t}^{\varepsilon}$, i.e., far from the singularity of $u_{\kappa}(t)$, while in [19, Proof of Lemma 5.5] this is done on $\Omega_{c t}$, where the product $\nabla \dot{u}_{\kappa}(t)$ is not in $L^{2}$ and $\nabla u_{\kappa}(t) \nabla \dot{u}_{\kappa}(t)$ is not integrable.

Concluding, if $\kappa=2 / \sqrt{\pi}$ and the Dirichlet boundary condition $w_{\kappa}$ is given by (4.3), then the growing crack corresponding to $s(t)=c t$ satisfies the dynamic energy-dissipation balance. This shows that, in this case, the stationary crack corresponding to $s(t)=0$ does not satisfy the $\eta$-maximal dissipation condition.

\section{Conclusions}

Dynamic evolutions are particularly problematic, due to the difficulty of showing energy balance, as well as of showing that solutions obey a maximal dissipation principle, or some similar condition that prevents stationary cracks from always being solutions.

We propose a quantitative version of a maximal dissipation principle as a selection criterion among all crack evolutions which satisfy the dynamic energy-dissipation balance. This principle, called $\eta$-maximal dissipation, depends on a threshold $\eta>0$, which can be interpreted as a characteristic length associated with the material.

In our main result, in Section 3, we make precise the hypotheses which guarantee the existence of an $\eta$-maximal dissipation solution, for any prescribed initial and boundary conditions.

It is well known that a stationary crack always satisfies the dynamic energy-dissipation balance. The example discussed in Section 4 shows that, in some cases, a stationary crack does not satisfy the $\eta$-maximal dissipation condition. In these cases the proposed selection criterion rules out stationary cracks.

ACKnowledgements. This material is based on work supported by the Italian Ministry of Education, University, and Research under the Project "Calculus of Variations" (PRIN 201011), by the European Research Council under Grant No. 290888 "Quasistatic and Dynamic Evolution Problems in Plasticity and Fracture", and by the National Science Foundation under Grant No. DMS-1313136. The first and third authors are members of the Gruppo Nazionale per l'Analisi Matematica, la Probabilità e le loro Applicazioni (GNAMPA) of the Istituto Nazionale di Alta Matematica (INdAM).

The authors wish to thank the referees for their useful comments, that contributed to improve the presentation of the results.

\section{REFERENCES}

[1] Anderson T.L.: Fracture Mechanics: Fundamentals and Applications, Third Edition 3rd Edition. Taylor \& Francis, Boca Raton, 2005

[2] Bouchbinder E., Fineberg J., Marder M.P. : Dynamics of simple cracks. Annu. Rev. Condens. Matter Phys. 1 (2010), 371-395.

[3] Bourdin B., Francfort G.A., Marigo J.-J.: The Variational Approach to Fracture. Springer, New York, 2008.

[4] Bourdin B., Larsen C.J., Richardson C.: A time-discrete model for dynamic fracture based on crack regularization. Int. J. Fracture 168 (2011), 133-143.

[5] Broberg K.B.: Cracks and Fracture. Academic Press, Cambridge, 1999.

[6] Dal Maso G., Larsen C.J.: Existence for wave equations on domains with arbitrary growing cracks. Atti Accad. Naz. Lincei Cl. Sci. Fis. Mat. Natur. Rend. Lincei (9) Mat. Appl. 22 (2011), 387-408. 
[7] Dal Maso G., Lucardesi I.: The wave equation on domains with cracks growing on a prescribed path: existence, uniqueness, and continuous dependence on the data. Preprint SISSA, Trieste, 2015.

[8] Dal Maso G., Scala R.: Quasistatic evolution in perfect plasticity as limit of dynamic processes. J. Dynam. Differential Equations 26 (2014), 915-954.

[9] Evans L.C.: Partial Differential Equations. Second edition. Graduate Studies in Mathematics, 19. American Mathematical Society, Providence, RI, 2010.

[10] Freund L.B.: Dynamic Fracture Mechanics. Cambridge Univ. Press, New York, 1990.

[11] Griffith A.: The phenomena of rupture and flow in solids. Philos. Trans. Roy. Soc. London Ser. A 221 (1920), 163-198.

[12] Haslach H.W.Jr.: Maximum Dissipation Non-Equilibrium Thermodynamics and its Geometric Structure. Springer, New York, 2011.

[13] Hofacker M., Miehe C.: A phase field model of dynamic fracture: robust field updates for the analysis of complex crack patterns. Internat. J. Numer. Methods Engrg. 93 (2013), 276-301.

[14] Irwin, G.R.: Analysis of stresses and strains near the end of a crack traversing a plate. J. Appl. Mech. 24 (1957), 361-364.

[15] Larsen C.J.: Models for dynamic fracture based on Griffith's criterion, in IUTAM Symp. Variational Concepts with Applications to the Mechanics of Materials, ed. K. Hackl, 131-140, Springer, Berlin, 2010

[16] Larsen C.J., Ortner C., Süli E.: Existence of solutions to a regularized model of dynamic fracture. Math. Models Methods Appl. Sci. 20 (2010), 1021-1048.

[17] Morozov N., Petrov Y.: Dynamics of Fracture. Foundations of Engineering Mechanics. Springer-Verlag, Berlin, 2000.

[18] Mott N.F.: Brittle fracture in mild steel plates, Engineering 165 (1948), 16-18.

[19] Nicaise S., Sändig A.-M.: Dynamic crack propagation in a 2D elastic body: the out-of-plane case. $J$ Math. Anal. Appl. 329 (2007), 1-30.

[20] Ravi-Chandar K.: Dynamic Fracture. Elsevier, Oxford,, 2004.

[21] Slepyan Y.: Models and Phenomena in Fracture Mechanics. Foundations of Engineering Mechanics. Springer-Verlag, Berlin, 2002.

[22] Zehnder A.T.: Fracture mechanics. Lecture Notes in Applied and Computational Mechanics, 62. Springer, London, 2012.

(Gianni Dal Maso) SISSA, Via Bonomea 265, 34136 Trieste, Italy

E-mail address, Gianni Dal Maso: dalmaso@sissa.it

(Christopher J. Larsen) Department of Mathematical Sciences, Worcester Polytechnic InstiTUte, 100 Institute RoAd, Worcester MA 01609-2280, USA

E-mail address, Christopher J. Larsen: cjlarsen@wpi.edu

(Rodica Toader) Dima, Università degli Studi di Udine, Via delle Scienze 206, 33100 Udine, Italy

E-mail address, Rodica Toader: rodica.toader@uniud.it 